\title{
Alveolar bone loss around mandibular anterior teeth in Class I, II, and III malocclusions: a CBCT evaluation study
}

\section{Zixiao Wang}

Shanghai Jiao Tong University

\section{Zhigui Ma}

Shanghai 9th People's Hospital

Chi Yang ( $\sim$ yangchi1963@hotmail.com )

Research article

Keywords: Alveolar bone dimension, Malocclusions, CBCT

Posted Date: June 19th, 2020

DOl: https://doi.org/10.21203/rs.3.rs-34913/v1

License: (9) (i) This work is licensed under a Creative Commons Attribution 4.0 International License. Read Full License 


\section{Abstract}

Background: To quantitatively and qualitatively evaluate alveolar defects of mandibular anterior alveolar bone in three different types of malocclusion with cone-beam computed tomography (CBCT).

Methods: A total of 542 teeth from 30 skeletal class I malocclusion (mean age: $19.26 \pm 5.63$ ), 33 class II malocclusion (mean age: $19.36 \pm 4.17$ ), and 28 class III malocclusion (mean age: $21.47 \pm 4.53$ ) patients were evaluated with CBCT. Sagittal sectional views were evaluated with regard to labial and lingual alveolar bone thickness and vertical alveolar bone level. Analysis of variance and Tukey's test were used for statistical comparisons at $\mathrm{P}<.05$

Results: Qualitative analysis: Class II (64.47\%) and Class III malocclusions (58.43\%) had higher prevalence of dehiscence than Class I malocclusions (32.96\%), $P<0.05$. No significant difference was found in the prevalence of fenestration among the three groups.

Quantitative analysis: In general, Class II and III groups had lesser alveolar bone volume than the Class I group. Furthermore, the vertical alveolar height and coronal alveolar bone thickness in Class II malocclusion was significantly lesser than that in the Class III group.

Conclusions: The Class II group, followed by the class III group, showed the most severe alveolar bone deficiency For these patients undergoing orthodontic treatment, special care should be taken to avoid aggravated preexisting alveolar bone loss in the mandibular anterior teeth.

\section{Background}

Orthodontic treatment is achieved by moving the teeth into the designed position along with bone remodeling. As the roots move away from the center of the alveolar bone, if the balance between resorption and apposition of the alveolar bone is not established, two stress concentration areas (the apical and alveolar crest parts) are at high risk of aggravated alveolar defects. Moreover, the reduced volume of the alveolar bone might change the center of tooth resistance during tooth movement, which result in further loss of alveolar bone. Previous studies showed that clinicians should be aware of the probability of thin cortical bone plates in patients suffering from insufficient alveolar bone (1). Periodontal problems such as bony dehiscence, fenestration, and gingival recession are often encountered, especially in the mandibular anterior teeth (2).

Few studies have reported that some certain types of malocclusions were associated with greater bone loss than that seen in normal occlusion samples. Enhos et al. (3) found that the incidence of dehiscence in patients with average angle and high angle was significantly higher than that in patients with low angle in their retrospective study. Higher prevalence of dehiscence and fenestration were found on the buccal or labial sides in all facial types. Nimigean et al. (4) suggested that tooth protrusion, tooth/jaw ratio, abnormal tooth position, and orthodontic tooth movement were considered as pathogenic factors 
for fenestration and dehiscence. To avoid these problems, the alveolar morphology should be determined before commencing orthodontic treatment.

Study (5) had shown that CBCT has high accuracy and repeatability in the linear measurement of alveolar bone, which could reflect the actual situation of alveolar bone topography and anatomy in anterior teeth. Three-dimensional (3D) analysis of specific regions of interest using best available tool for identifying these bone-supporting periodontal conditions.

To the authors' best knowledge, no study has yet compared the alveolar bone status in subjects with Class I, Class II, and Class III malocclusion, with both quantitative and qualitative assessments.

Therefore, the purpose of this study is to investigate the amount of alveolar bone loss and thickness around the mandibular anterior teeth of Chinese patients with various types of malocclusion, and further provide guidance in routine treatment planning. The null hypothesis is that there is no difference among these three groups.

\section{Methods}

\subsection{Study design}

A cross-sectional clinical study was approved by the Institutional Review Board at the Ninth People's Hospital (Shanghai, China). From November 2013 to December 2015, a consecutive series of patients included in this study were considered based on the following inclusion criteria: (1) age $\geq 18$ years; (2) Class I malocclusion (ANB $\left.=0-4^{\circ}\right)$, Class II malocclusion (ANB $\left.>4^{\circ}\right)$, and Class III malocclusion $\left(A N B<0^{\circ}\right)$ (Dolphin software v11.7, Chatsworth, CA, USA); (3) mild anterior teeth crowding (0-4 mm); and (4) clinical and CBCT examination showing potential dehiscence and fenestration or thin alveolus and prominent root(s) in the anterior region. The exclusion criteria included: (1) history of trauma or surgery; (2) history of orthodontic or orthognathic treatment; (3) active periodontal disease; (4) abnormal tooth shape or restoration of lower anterior teeth; (5) congenital craniofacial malformation syndrome; and (6) smoking history. The basic information of patients were presented in Table 1 and Table 2.

Image acquisition of measurement method

CBCT scans were obtained using Kodak digital oral CT (Kodak, 9000C 3DX Eastman Kodak Company). The scan range included the mandibular dentition, from left mandibular second molar to right mandibular second molar including the complete root. The scanning method comprised Triple beam technology (front and right beams, left and right beams, upper and lower beams) were located, ensuring identical head position of different patients. The scanning parameters included ball tube voltage $70 \mathrm{kV}$ and current $8 \mathrm{~mA}$, and the scanning conditions were scanning time $10.68 \mathrm{~s}$ and thickness $0.2 \mathrm{~mm}$. All lateral cephalograms were obtained from the original CBCT data.

\subsection{Measurement index}




\subsubsection{Evaluation index of bone dehiscence/bone fenestration}

According to the study by Evangelisa et al. (6), bone defect was classified as dehiscence when the alveolar bone height was more than $2 \mathrm{~mm}$ from the cementoenamel junction. If the absence of cortical bone around the root did not involve the alveolar crest, it was classified as fenestration.

\subsubsection{Measurement}

CBCT images and lateral cephalograms were imported into the Dolphin software in DICOM format, and $3 \mathrm{D}$ reconstructions were performed. According to our previous measurement method (7), the largest labiolingual section of the mandibular central incisors, lateral incisors, and canines was chosen as the measurement plane (Fig. 1). Horizontal bone thickness was recorded perpendicular to the long axis of each lower anterior tooth. Labial and lingual bone thickness measurements were made from the midpoint of the coronal third (ACHBT/PCHBT), middle third (AMHBT/PMHBT), and apical third (AAHBT/PAHBT) to the limit of the labial cortical surfaces. Per our previous report (7), the definitions of all landmarks and measurement variables used in this study are presented in Table 3. Digital tracings of lateral cephalograms were also performed using the Dolphin software.

\subsection{Statistical analysis}

All tracing and metrical assessment was performed by one investigator, the measurements were repeated two weeks later by the same operator, and the average value was taken as the measured data.

A $\chi^{2}$ test was also performed using SPSS 16.0 (Chicago, IL) for analyzing the frequency analysis. Oneway analysis of variance (ANOVA) and the LSD test were used for comparisons of alveolar defects in different malocclusion groups. A confidence level of $\mathrm{P}<.05$ was considered to indicate statistical significance.

\section{Results}

This study included 542 teeth from 30 subjects with Class I malocclusion ( 17 male and 13 female, mean age: $19.26 \pm 5.63$ years); 33 with Class II malocclusion ( 15 male and 18 female, mean age: $19.36 \pm$ 4.17 years); and 28 with Class III malocclusion (14 male and 14 female, mean age: $21.47 \pm 4.53$ years).

\subsection{Alveolar fracture/bone fenestration in all types of malocclusions}

The results are shown in Table 4.

The Class II and III malocclusion groups showed greater prevalence of dehiscence than the Class I group; no significant difference was found in the incidence of fenestration among the three groups. 
Furthermore, root exposure (dehiscence and fenestration) appeared more in canines (Class I, 45.76\%; Class II, 96.97\%, Class III, 71.43\%, respectively) than other teeth in all groups.

\subsection{Alveolar measurements in different malocclusions}

Tables 5-7 show the buccal and lingual alveolar measurements for the lower anterior teeth.

Mandibular central incisor: The thickness of alveolar bone in the upper 1/3, middle $1 / 3$ of the labial side, and middle $1 / 3$ of the lingual side of the root in Class II malocclusion were significantly lower than those in Class I malocclusion $(\mathrm{P}<.05)$. The height of labial and lingual alveolar bone in Class II malocclusion was less than that in Class I malocclusion $(P<.05)$. The width of the labial alveolar bone in the middle $1 / 3$ of the root in Class II malocclusion was less than that observed in Class I malocclusion $(P<.05)$. The vertical height of the lingual alveolar bone in patients with class III malocclusion was significantly lower than that of patients with class II malocclusion $(P<.05)$ (Table 5).

Mandibular lateral incisor: Except for the thickness of alveolar bone in the middle 1/3 of the labial side, all other measurements showed that the alveolar bone of class II malocclusions was thinner than that of Class I malocclusion $(P<.05)$. The vertical height of alveolar bone of lower incisor was significantly less in Class II malocclusion $(\mathrm{P}<.01)$. The horizontal alveolar bone thickness of the coronal third of the labial side and the labial vertical alveolar bone in Class III malocclusion were significantly lesser than those in in Class I malocclusion $(P<.05)$. Further, the lingual horizontal alveolar bone thickness of the coronal third and the lingual vertical alveolar bone level in Class II malocclusion was significantly lesser than those in Class III malocclusion $(\mathrm{P}<.01)$ (Table 6).

Mandibular canine: The thickness of the lingual alveolar bone at the coronal third, labial alveolar bone at apical third, and lingual vertical alveolar bone level in Class II and III malocclusions were lesser than those in Class I malocclusion $(P<.01)$. Additionally, the width of the buccal alveolar bone at the middle third and labial vertical alveolar bone height in Class II malocclusion were less than those seen in Class I malocclusion $(P<.05)$. The labial bone thickness at the coronal third and labial alveolar bone level was significantly lesser in Class II malocclusion than in Class III malocclusion ( $<$.05) (Table 7).

\section{Discussion}

The null hypothesis was rejected as to say that there were differences in all three groups. In this study, 542 mandibular anterior teeth from 91 samples were included, and CBCT was used to evaluate the alveolar bone defects and morphology of mandibular anterior teeth. However, quantitative measurements of vertical height and horizontal width of the alveolar bone with fenestration and dehiscence have been rarely reported in previous literature.

Various studies have shown thin bone structure in the lower anterior incisors, and orthodontic treatments present a high risk for moving the lower anterior teeth in the labiolingual direction, especially in case of mandibular prognathism (8-10). However, in the current study, we found that Class II subjects had more alveolar defects than Class I and Class III subjects. Compensation of the mandibular anterior teeth may 
be a crucial factor that leads to inadequate alveolar bone volume in the lower anterior region. Thus, decompensation of anterior teeth during orthodontic treatment that exceeds the alveolar housing is associated with gingival recession or alveolar bone loss.

In this study, we found that the incidence of bone fenestration and bone dehiscence was most likely to occur in Class II malocclusion, followed by Class III and Class I malocclusion. Similarly, Yagci et al. (11) also found that the incidence of fenestration in Class II malocclusions was significantly higher than that seen in Class I or III malocclusions.

Nahm et al. (12) investigated the CBCT data of 24 patients with Class I occlusion with bimaxillary protrusion and found that there was insufficient bone support in the incisor region, especially in the lingual side. However, in our study, the insufficiency of labial bone was more pronounced. Evangelista et al. (6) analyzed 4319 teeth to compare the incidence of alveolar bone defect in Class I and Class II division 1 malocclusions and found that incidence of bone dehiscence in Class I malocclusion was 35\% higher than that in Class II malocclusion, which was inconsistent with our results.

The bone defect may be related to the craniofacial vertical growth pattern, which affects the morphology of the labiolingual alveolar bone cortex (3). Various studies have shown that patients with hyperdivergent pattern have thinner and narrower alveolar bone, especially in the anterior mandibular region, than those with hypo-divergent pattern $(13,14)$. A possible reason is that a hyperdivergent pattern increases the risk, as it is accompanied by a thin symphysis and vertical elongation of the incisors (15), increasing the likelihood of periodontal side effects. In the current study, the average mandibular angle of Class II malocclusion was $34.99^{\circ}$, which was higher than that of Class I $\left(26.61^{\circ}\right)$ and Class III $\left(31.33^{\circ}\right)$ malocclusion. This also proves that the alveolar bone defect that was more predominant in Class II malocclusion may be related to vertical growth type, although Evangelista et al. (6) suggested that vertical growth type has no effect on alveolar defect.

Many studies only evaluate the alveolar bone around incisors. However, Nimigean et al. (4) noted that the mandibular canines are high risk sites of dehiscence in 138 dry skulls. We speculated whether the alveolar bone morphology of canines was similar between incisors and canines. Therefore, in this present study, we focused on the alveolus surrounding the lower anterior teeth including the canines in crosssectional slices at the buccal and lingual surfaces. Our results showed that fenestration occurred more frequently in the mandibular canines. One possible reason was that the lower canine teeth were located at the corner of the arch, leading to a high tendency for this defect. Mandibular central incisors were most likely to exhibit bony dehiscence instead of bony fenestration. This is positively correlated with the anatomical characteristics and position of the mandibular central incisor, which is located at the symphysis where congenital thinner bone cortex may develop $(16,17)$. Our study results were consistent with the findings of Evangelista et al., who reported that dehiscence occurred with greater frequency in the lower central incisor. Furthermore, in our future study, we aim to classify the fenestration and dehiscence of each anterior tooth and study the alveolar bone change around anterior teeth during orthodontic treatment. 
Compared with Class I malocclusion, Class II and III malocclusions showed significantly reduced vertical alveolar bone level of labiolingual aspects. Per clinical observation, it appears that the occurrence of labial alveolar bone loss and gingival recession is more common during surgical orthodontic treatment in patients with Class III malocclusion (18). Moreover, the vertical height of alveolar bone of Class II malocclusion was significantly lower than that of Class III malocclusion by the lingual and labial side of the central incisor, the lateral incisor, and the labial side of the canine. The possible reason may be related to dental compensation. Anatomically, the mandibular anterior teeth tended to be proclined to the labial side in patients with Class II malocclusion (19). Overall, either Class II or Class III malocclusions could result in the reduction of vertical alveolar bone level, especially on the labial cortical side.

CBCT should be performed to observe the alveolar bone defect before orthodontic treatment, especially for patients with Class II and III malocclusions. Previous studies (20-23) have reported that once the cortical plate had been penetrated by the root, recovery of the well-defined dense cortical plate would not occur. The potential of developing fenestrations and dehiscences must be carefully evaluated through orthodontic tooth movement. This study attempts to help the clinician design and manage treatment to clinically change the magnitude and direction of the force and bone augmentation if necessary, such that alveolar bone integrity during orthodontic treatment can be maintained as much as possible. Future studies are needed to confirm the etiology of alveolar bone defect and anatomic boundary of tooth movement.

Conclusions:

Dehiscence was encountered more frequently in Class II and Class III malocclusions. In addition, the loss of vertical alveolar bone and coronal alveolar bone thickness was greater in the Class II malocclusion than in Class III. Special care should be taken to avoid acceleration of preexisting bone loss during orthodontic treatment.

\section{Abbreviations:}

CEJ, Cementoenamel junction, respectively.

ACHBT\&PCHBT, horizontal bone thickness at the middle level of the coronal third of the buccal and lingual side, respectively; AMHBT\&PMHBT, horizontal bone thickness at the middle level of the middle third of the labial and lingual side, respectively. AAHBT\& PAHBT, horizontal bone thickness at the middle level of the apical third of the labial and lingual side, respectively. AVBL\& PVBL, vertical alveolar bone level at the labial and lingual side, respectively.

\section{Declarations}

Ethics approval and consent to participate 
The project was proved by Scientific Research Projects Approval of Independent Ethics Committee of Shanghai Ninth People's Hospital affiliated to ShanghaiJiaoTongUniversity, School of Medicine. Number: 2017-368

Informed consent (written version) was obtained from all participants.

Consent for publication

Not applicable.

Availability of data and material

The datasets used and/or analysed during the current study are available from the corresponding author on reasonable request.

Competing interests

The authors declare that they have no competing interests.

\section{Funding}

Not applicable.

\section{Author's contributions}

Dr. Zixiao Wang participated in the study design, acquisition of data, analysis and interpretation of data, drafting and revising the manuscript for important intellectual content together. Prof. Chi Yang \& Zhigui Ma participated in the study design, reviewed and revised the manuscript, and approved the final manuscript as submitted. All authors approved the final manuscript as submitted and agree to be accountable for all aspects of the work.

Acknowledgements

Not applicable.

\section{References}

1. Ma ZG, Yang C, Fang B, Xia YH, Mao LX, Feng YM. Three-D imaging of dental alveolar bone change after fixed orthodontic treatment in patients with periodontitis. Int J Clin Exp Med. 2015;8(2):238591.

2. Horiuchi A, Hotokezaka H, Kobayashi K. Correlation between cortical plate proximity and apical root resorption. Am J Orthod Dentofacial Orthop. 1998;114(3):311-8.

3. Enhos S, Uysal T, Yagci A, Veli I, Ucar Fl, Ozer T. Dehiscence and fenestration in patients with different vertical growth patterns assessed with cone-beam computed tomography. Angle Orthod. 
2012;82(5):868-74. doi:10.2319/111211-702.1.

4. Nimigean VR, Nimigean V, Bencze MA, Dimcevici-Poesina N, Cergan R, Moraru S. Alveolar bone dehiscences and fenestrations: an anatomical study and review. Rom J Morphol Embryol. 2009;50(3):391-7.

5. Lou L, Lagravere MO, Compton S, Major PW, Flores-Mir C. Accuracy of measurements and reliability of landmark identification with computed tomography (CT) techniques in the maxillofacial area: a systematic review. Oral Surg Oral Med Oral Pathol Oral Radiol Endod. 2007;104(3):402-11. doi:10.1016/j.tripleo.2006.07.015.

6. Evangelista K, Vasconcelos Kde F, Bumann A, Hirsch E, Nitka M, Silva MA. Dehiscence and fenestration in patients with Class I and Class II Division 1 malocclusion assessed with cone-beam computed tomography. Am J Orthod Dentofacial Orthop. 2010;138(2):133.

doi:10.1016/j.ajodo.2010.02.021. e1-7; discussion - 5.

7. Ma ZG, Yang C, Xie QY, Ye ZX, Zhang SY, Abdelrehem A. A Novel Surgical Technique for Augmented Corticotomy-Assisted Orthodontics: Bone Grafting With Periosteum. J Oral Maxillofac Surg. 2016;74(1):170-80. doi:10.1016/j.joms.2015.06.147.

8. Ahn HW, Lee DY, Park YG, Kim SH, Chung KR, Nelson G. Accelerated decompensation of mandibular incisors in surgical skeletal class III patients by using augmented corticotomy: a preliminary study. Am J Orthod Dentofacial Orthop. 2012;142(2):199-206. doi:10.1016/j.ajodo.2012.03.028.

9. Kim Y, Park JU, Kook YA. Alveolar bone loss around incisors in surgical skeletal Class III patients. Angle Orthod. 2009;79(4):676-82. doi:10.2319/070308-341.1.

10. Kook YA, Kim G, Kim Y. Comparison of alveolar bone loss around incisors in normal occlusion samples and surgical skeletal class III patients. Angle Orthod. 2012;82(4):645-52. doi:10.2319/070111-424.1.

11. Yagci A, Veli I, Uysal T, Ucar FI, Ozer T, Enhos S. Dehiscence and fenestration in skeletal Class I, II, and III malocclusions assessed with cone-beam computed tomography. Angle Orthod. 2012;82(1):67-74. doi:10.2319/040811-250.1.

12. Nahm KY, Kang JH, Moon SC, et al. Alveolar bone loss around incisors in Class I bidentoalveolar protrusion patients: a retrospective three-dimensional cone beam CT study. Dentomaxillofac Radiol. 2012;41(6):481-8. doi:10.1259/dmfr/30845402.

13. Handelman CS. The anterior alveolus: its importance in limiting orthodontic treatment and its influence on the occurrence of iatrogenic sequelae. Angle Orthod. 1996;66(2):95-109; discussion 10. doi:10.1043/0003-3219(1996)066<0095:TAAIII>2.3.C0;2

14. Horner KA, Behrents RG, Kim KB, Buschang PH. Cortical bone and ridge thickness of hyperdivergent and hypodivergent adults. Am J Orthod Dentofacial Orthop. 2012;142(2):170-8. doi:10.1016/j.ajodo.2012.03.021.

15. Ye R, Li Y, Li X, et al. Occlusal plane canting reduction accompanies mandibular counterclockwise rotation in camouflaging treatment of hyperdivergent skeletal Class II malocclusion. Angle Orthod. 2013;83(5):758-65. doi:10.2319/101512-801.1. 
16. Blanco J, Alonso A, Sanz M. Long-term results and survival rate of implants treated with guided bone regeneration: a 5-year case series prospective study. Clin Oral Implants Res. 2005;16(3):294-301. doi:10.1111/j.1600-0501.2005.01106.x.

17. Rupprecht RD, Horning GM, Nicoll BK, Cohen ME. Prevalence of dehiscences and fenestrations in modern American skulls. J Periodontol. 2001;72(6):722-9. doi:10.1902/jop.2001.72.6.722.

18. Albandar JM, Rise J, Gjermo P, Johansen JR. Radiographic quantification of alveolar bone level changes. A 2-year longitudinal study in man. J Clin Periodontol. 1986;13(3):195-200.

19. Freitas MR, Santos MA, Freitas KM, Janson G, Freitas DS, Henriques JF. Cephalometric characterization of skeletal Class II, division 1 malocclusion in white Brazilian subjects. J Appl Oral Sci. 2005;13(2):198-203. doi:10.1590/s1678-77572005000200020.

20. Wainwright WM. Faciolingual tooth movement: its influence on the root and cortical plate. Am J Orthod. 1973;64(3):278-302.

21. Sarikaya S, Haydar B, Ciger S, Ariyurek M. Changes in alveolar bone thickness due to retraction of anterior teeth. Am J Orthod Dentofacial Orthop. 2002;122(1):15-26.

22. Duterloo HS. [Orthodontic treatment and remodeling of the bone tissue of the alveolar process (proceedings)]. Rev Belge Med Dent. 1977;32(4):396.

23. Remmelink HJ, van der Molen AL. Effects of anteroposterior incisor repositioning on the root and cortical plate: a follow-up study. J Clin Orthod. 1984;18(1):42-9.

\section{Tables}

Table 1 Distribution of included subjects

\begin{tabular}{|lllllllllll|}
\hline & I & \multicolumn{1}{c}{ II } & \multicolumn{7}{c|}{ III } \\
\cline { 2 - 5 } & Male & Female & Total & Male & Female & Total & Male & Female & Total \\
$\begin{array}{l}\text { Number of } \\
\text { patients }\end{array}$ & 17 & 13 & 30 & 15 & 18 & 33 & 14 & 14 & 28 \\
\hline Number of teeth & 102 & 77 & 179 & 89 & 108 & 197 & 84 & 82 & 166 \\
\hline Central incisors & 34 & 26 & 60 & 30 & 36 & 66 & 28 & 26 & 54 \\
\hline Lateral incisors & 34 & 26 & 60 & 29 & 36 & 65 & 28 & 28 & 56 \\
\hline Canines & 34 & 25 & 59 & 30 & 36 & 66 & 28 & 28 & 56 \\
\hline
\end{tabular}

Table 2 Demographic data of patients with Class I, Class II, and Class III malocclusions 


\begin{tabular}{|c|c|c|c|c|c|c|}
\hline \multirow[t]{2}{*}{ Parameters } & \multicolumn{2}{|l|}{ I } & \multicolumn{2}{|l|}{ II } & \multicolumn{2}{|l|}{ III } \\
\hline & Mean & SD & Mean & SD & Mean & SD \\
\hline 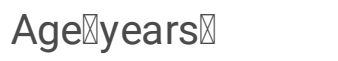 & 19.26 & 5.63 & 19.36 & 4.17 & 21.47 & 4.53 \\
\hline SNA $\mathbb{Z}^{\circ} \mathbb{Z}$ & 82.46 & 2.20 & 82.11 & 2.96 & 79.28 & 1.12 \\
\hline SNB $\mathbb{D}^{\circ} \rrbracket$ & 78.97 & 4.46 & 75.46 & 5.73 & 80.77 & 3.25 \\
\hline $\mathrm{ANB} \mathbb{\nabla}^{\circ} \rrbracket$ & 3.49 & 0.81 & 6.65 & 1.54 & -1.49 & 1.07 \\
\hline Overjet (mm) & 2.03 & 1.44 & 5.82 & 2.91 & -1.43 & 1.55 \\
\hline 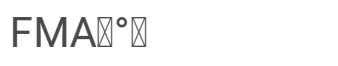 & 26.61 & 4.50 & 34.99 & 5.30 & 31.33 & 7.49 \\
\hline $\mathrm{FMIA}^{\circ} \rrbracket$ & 59.67 & 8.83 & 49.41 & 6.57 & 64.01 & 10.31 \\
\hline $\mathrm{IMPA}^{\circ} \mathbb{\nabla}$ & 93.48 & 8.87 & 95.89 & 7.45 & 84.27 & 6.81 \\
\hline U1-SN $\mathbb{Z}^{\circ} \mathbb{Z}$ & 104.23 & 8.99 & 102.22 & 7.79 & 107.93 & 6.81 \\
\hline $\begin{array}{l}\text { Interincisal angel } \\
\nabla^{\circ} \square\end{array}$ & 126.99 & 14.90 & 119.43 & 8.85 & 128.57 & 9.36 \\
\hline
\end{tabular}

Table 3 Definitions of linear measurements

\begin{tabular}{|c|c|}
\hline $\begin{array}{l}\text { Reference } \\
\text { points } \\
\text { and lines }\end{array}$ & Definition \\
\hline CEJ & Cementoenamel junction \\
\hline P1 & Alveolar crest at labial side \\
\hline P2 & Root exposure without involvement of alveolar crest by occlusional margin \\
\hline P3 & Root exposure without involvement of alveolar crest by gingival margin \\
\hline $\mathrm{d} \rrbracket \mathrm{mm} \rrbracket$ & $\begin{array}{l}\text { A line tangent to the long axis of the tooth and passing through } \mathrm{P} 1 \text { was made }(\mathrm{x} 1) \text {. Line } \\
(\mathrm{x} 2) \text { was parallel to }(\mathrm{x} 1) \text {, passing through the CEJ point at the labial side. The distance } \\
\text { between line }(\mathrm{x} 1) \text { and line }(\mathrm{x} 2) \text { is defined as } \mathrm{d} \text {. }\end{array}$ \\
\hline $\mathrm{f} \bigotimes \mathrm{mm} \rrbracket$ & $\begin{array}{l}\text { A line tangent to the long axis of the tooth and passing through } P 3 \text { was made }(x 3) \text {. Line } \\
(x 4) \text { was parallel to }(x 3) \text {, passing through } P 2 \text {. The distance between line }(x 3) \text { and line } \\
(x 4) \text { is defined as } f .\end{array}$ \\
\hline
\end{tabular}

Table 4 The prevalence of dehiscence and fenestration on the lower anterior region for different 


\begin{tabular}{|lllllllllll|}
\hline & \multicolumn{3}{l}{ Bony dehiscence } & \multicolumn{5}{c|}{ Bony fenestration } & \multicolumn{4}{c|}{$\begin{array}{c}\text { Dehiscence and } \\
\text { fenestration }\end{array}$} \\
\cline { 2 - 11 } & I & II & III & I & II & III & I & II & III \\
$\begin{array}{l}\text { Central } \\
\text { incisor }\end{array}$ & 33.33 & 74.24 & 70.37 & 5.00 & 4.55 & 7.41 & 38.33 & 78.79 & 77.78 \\
\hline $\begin{array}{l}\text { Lateral } \\
\text { incisor }\end{array}$ & 35.00 & 49.23 & 53.57 & 20.00 & 12.31 & 14.28 & 55.00 & 61.54 & 67.86 \\
\hline Canine & 30.50 & 69.70 & 51.79 & 15.25 & 27.27 & 19.64 & 45.76 & 96.97 & 71.43 \\
\hline Total & 32.96 & $64.47^{*}$ & $58.43^{\ddagger}$ & 13.41 & 14.72 & 13.86 & 46.37 & $79.19^{*}$ & $72.29^{\ddagger}$ \\
\hline
\end{tabular}

*P<.05 vs. Class I group;

${ }^{\ddagger} \mathrm{P}<0.1$ vs. Class I group.

Table 5 Alveolar measurements in different malocclusions for lower middle incisors

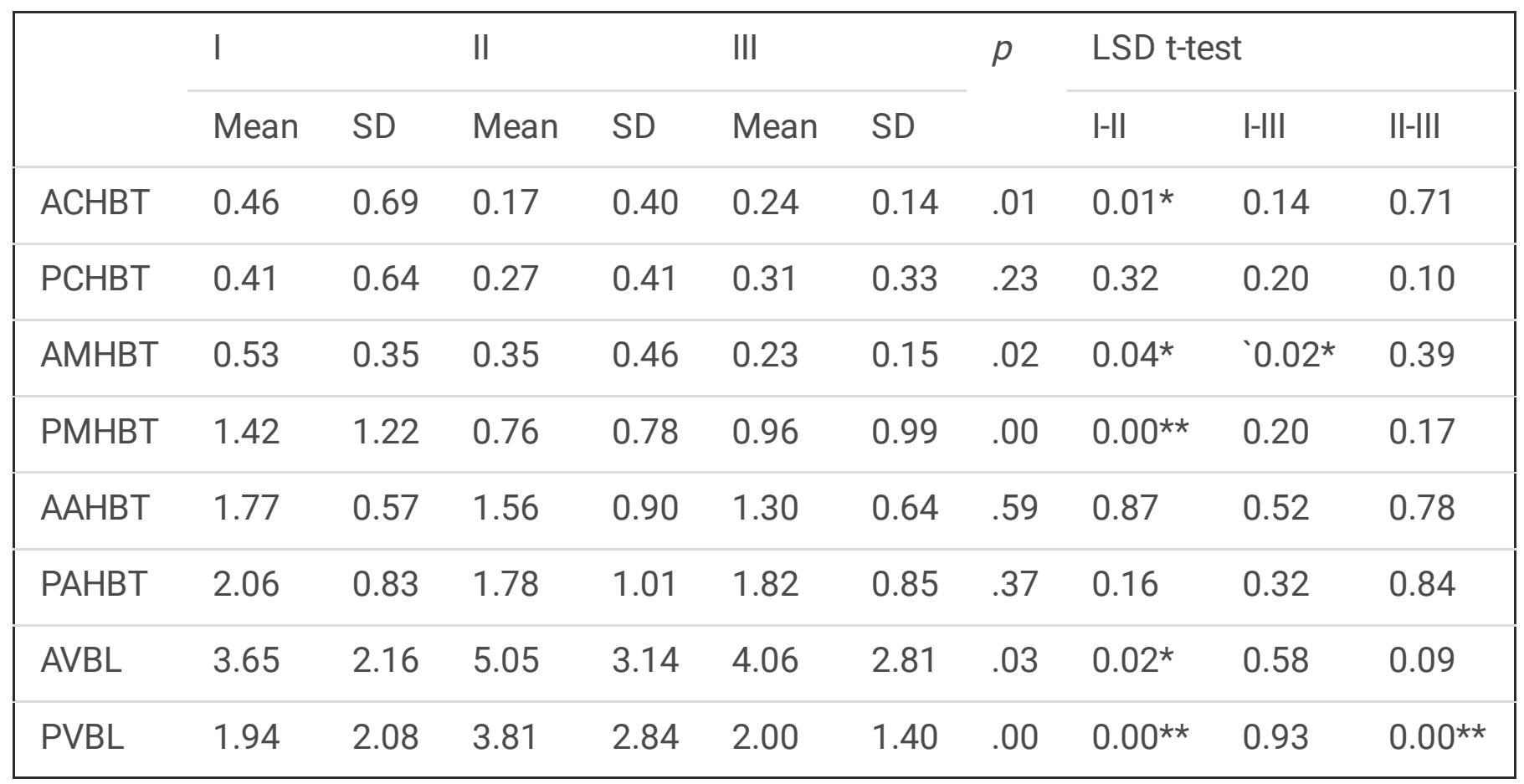

Abbreviations:

ACHBT\&PCHBT, horizontal bone thickness at the middle level of the coronal third of the buccal and lingual side, respectively; AMHBT\&PMHBT, horizontal bone thickness at the middle level of the middle third of the labial and lingual side, respectively. AAHBT\& PAHBT, horizontal bone thickness at the middle level of the apical third of the labial and lingual side, respectively. AVBL\& PVBL, vertical alveolar bone level at the labial and lingual side, respectively.

Table 6 Alveolar measurements in different malocclusions for lower lateral incisors 


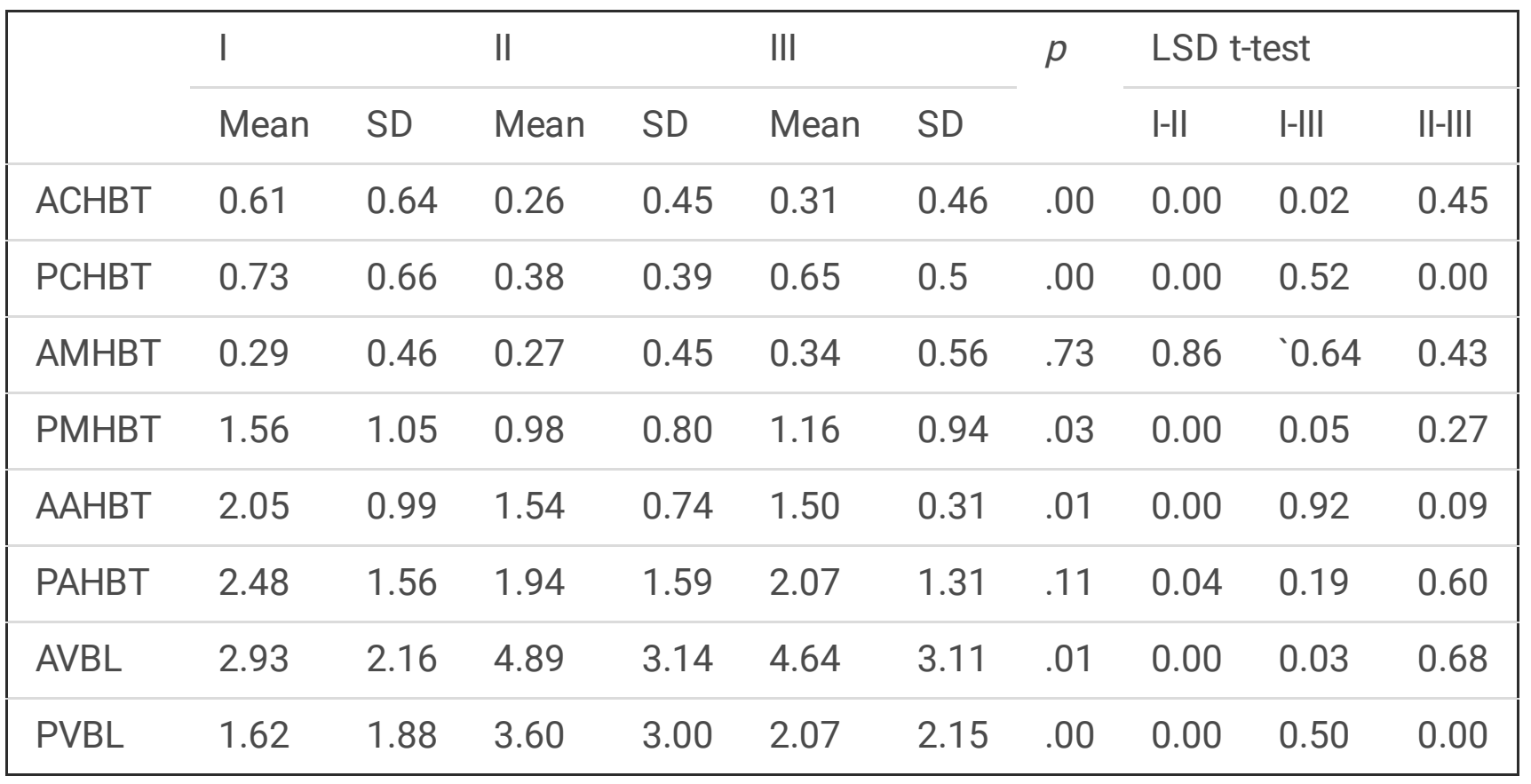

Table 7 Alveolar measurements in different malocclusions for lower canines

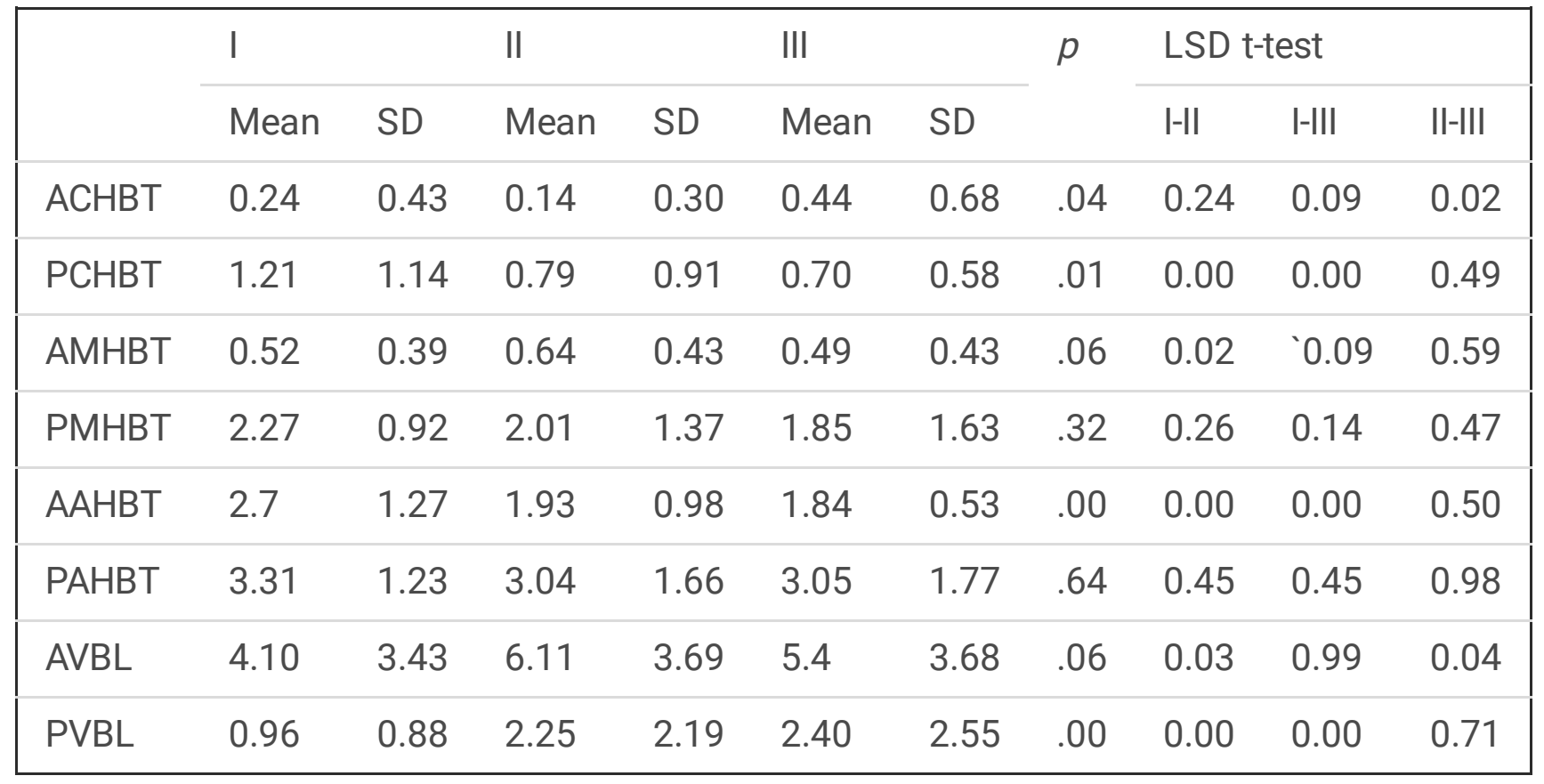

\section{Figures}



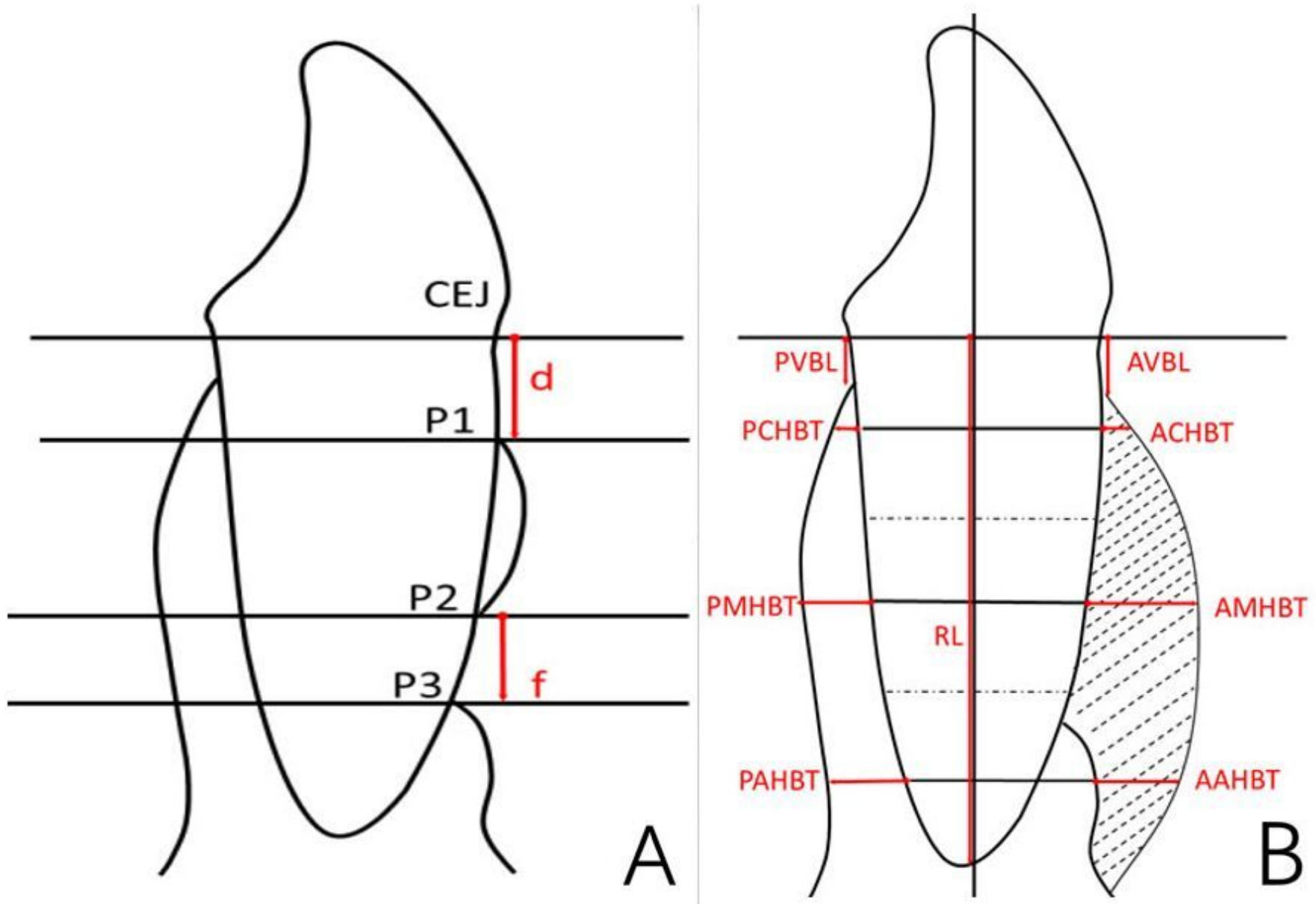

Figure 1

A Illustrations of reference points for dehiscence and fenestration; B Illustrations of reference points and measurement variables used in this study. 